\title{
Giant Ganglioneuroma of Thoracic Spine : A Case Report and Review of Literature
}

\author{
Yong Huang, ${ }^{1,2}$ Lidi Liu, M.D., ${ }^{1}$ Qiao Li, M.D., ${ }^{1}$ Shaokun Zhang, M.D., Ph.D. \\ Department of Orthopedics,' The First Hospital of Jilin University, Changchun, China \\ Department of Orthopedics, ${ }^{2}$ Taihe Hospital, Hubei University of Medicine, Shiyan, China
}

Ganglioneuroma (GN) is a rare benign tumor of neural crest origin usually found in the abdomen, but may occasionally present at uncommon sites including the cervical, lumbar, or sacral spine. However, GNs of thoracic spine are extremely rare. In this report, we describe a 12-year-old girl with giant GN in the thoracic spine, who underwent successful resection (T1-4 level) of the tumor. Histopathological examination confirmed the diagnosis. GN should be considered in the differential diagnosis of any paraspinal mass. A high index of suspicion and correlation of clinico-radiological findings is necessary in differentiating a large benign tumor from a malignant growth. Complete surgical excision is the treatment of choice; however tumor size and location need to be considered for the surgical approach (one-step or multiple surgeries). Close follow-up after surgery is mandatory.

Key Words : Ganglioneuroma · Intradural tumor · Thoracic spine · Surgery.

\section{INTRODUCTION}

Ganglioneuroma $(\mathrm{GN})$ is a rare benign tumor that originates from neural crest cells of sympathetic ganglia or adrenal medulla, and usually occur in the retroperitoneum (especially presacral space), posterior mediastinum, or adrenal gland, and occasionally in unusual locations. ${ }^{1,3,6,8)}$ We report a giant paraspinal GN extending into extradural space and thoracic cavity.

\section{CASE REPORT}

A 12-year-old girl presented with a one month history of sudden onset chest tightness and gradually increasing lower extremity weakness. The patient had no other significant complaints. On examination, she was unable to stand or walk without support. A firm paraspinal mass measuring $12 \times 12 \times$ $12 \mathrm{~cm}$ was seen at the upper thoracic vertebral level.

Magnetic resonance imaging showed a long intradural extramedullary tumor, extending from $\mathrm{T} 1$ to $\mathrm{T} 4$ levels. The tumor extended through the left T2/3, T3/4 neural foramen into the left thoracic cavity. The intervertebral foramen was enlarged. The lesion was mildly hypo-intense on T1-weighted imaging, and heterogeneously hyper-intense on T2 weighted imaging (Fig. 1). Surgery required a combined multidisciplinary team. A two-step surgical intervention was planned considering that a single stage surgery is potentially more complex, takes longer time, results in more bleeding, and is

- Received : August 4, 2015 •Revised : November 24, 2015 •Accepted : February 19, 2016

- Address for reprints : Shaokun Zhang, M.D., Ph.D.

Department of Orthopedics, The First Hospital of Jilin University, 71 Xinmin Street, Changchun, 130021, China

Tel : +86-15104302235, Fax : +86-0431-88785361, E-mail : haungyong18@163.com

This is an Open Access article distributed under the terms of the Creative Commons Attribution Non-Commercial License (http://creativecommons.org/licenses/by-nc/4.0) which permits unrestricted non-commercial use, distribution, and reproduction in any medium, provided the original work is properly cited. 


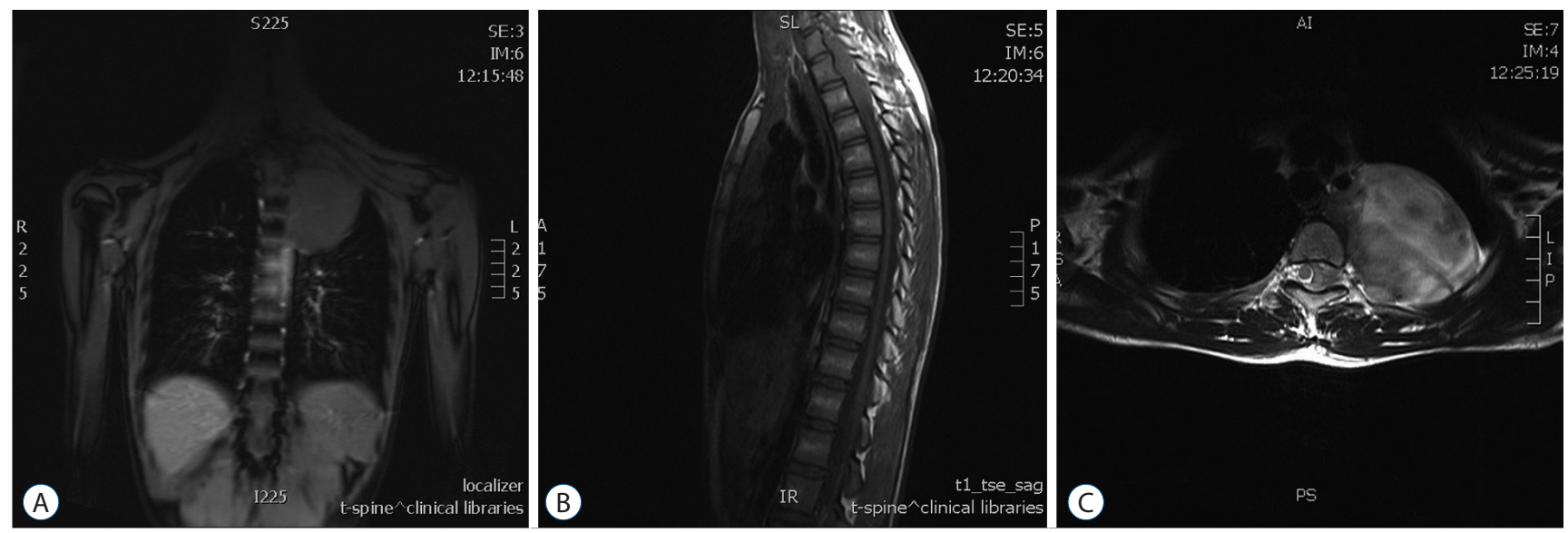

Fig. 1. Preoperative MRI. A : Coronal T1-weighted image showing a giant, mildly hypointense left-sided mass. B : Sagittal T1-weighted MRI showing a hypointense strip at the T1-T4 level. C : Axial T2-weighted MRI at the T3-4 level showing a heterogeneously hyper-intense mass extending to the spinal canal. MRI : magnetic resonance imaging.

usually performed in younger patient. The prognosis was explained to her parents.

\section{The first resection}

After performing the left hemilaminectomy with Cavitron Ultrasonic Surgical Aspirator (CUSA) and opening the dura from T1 to T4 levels, the cord was found to be severely compressed by the intradural tumor, which was totally resected under the microscope during surgery under careful neurophysiological monitoring which showed no obvious abnormalities. There was no neurodeficit after the first surgery.

\section{The second resection}

Two weeks later, a second resection was performed to remove the tumor infiltrating into the thoracic cavity. The tumor was found connected to the nerve roots, and had involved the pleura at left cupula. Subtotal resection for the foraminal portions was performed, and the left C3 nerve root was preserved. The intraoperative blood loss was about $200 \mathrm{~mL}$.

\section{Pathological examination}

Macroscopically, the tumor was smooth and well-encapsulated. On serial slicing, the tumor was firm and homogenous in appearance with no areas of hemorrhage, necrosis, or any signs of degeneration. Microscopically, the tumor was composed of mature ganglion cells, surrounded by schwann cells in a fibrillary background. On immunohistochemical evaluation, the tumor cells were positive S-100 protein and neuron

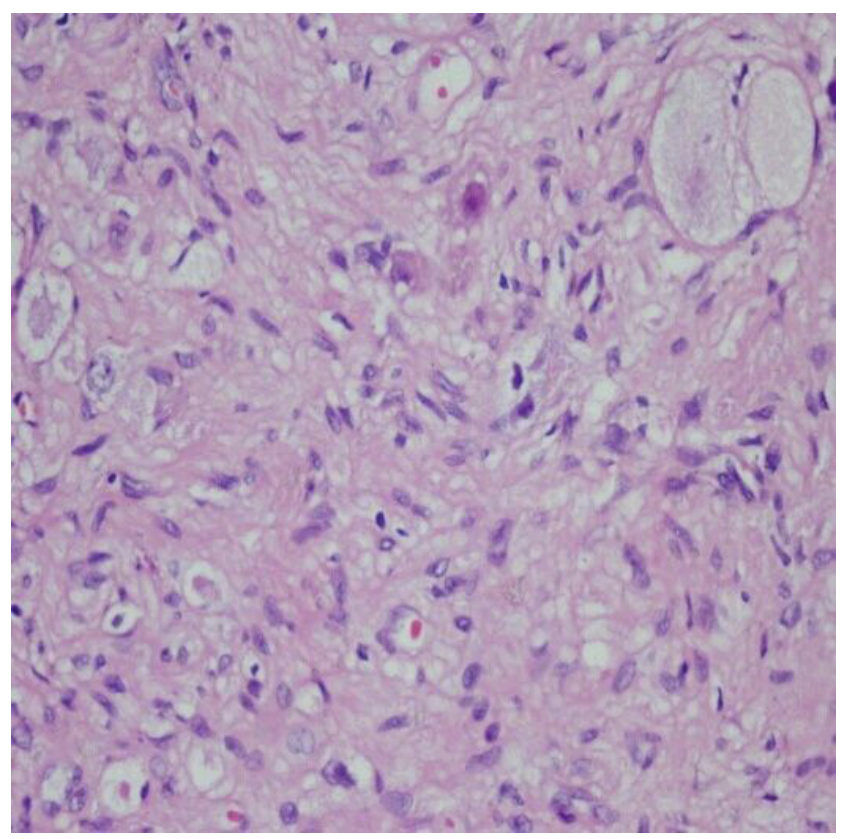

Fig. 2. H\&E stained section showing mature ganglion cells surrounded by schwann cells and fibrillary stroma (400x).

specific enolase, and the Ki-67 proliferation index was less than $1 \%$ (Fig. 2).

\section{Management}

Postoperative chest computed tomography confirmed volume reduction of tumor. The patient had an uneventful recovery in a one-year follow-up period. 


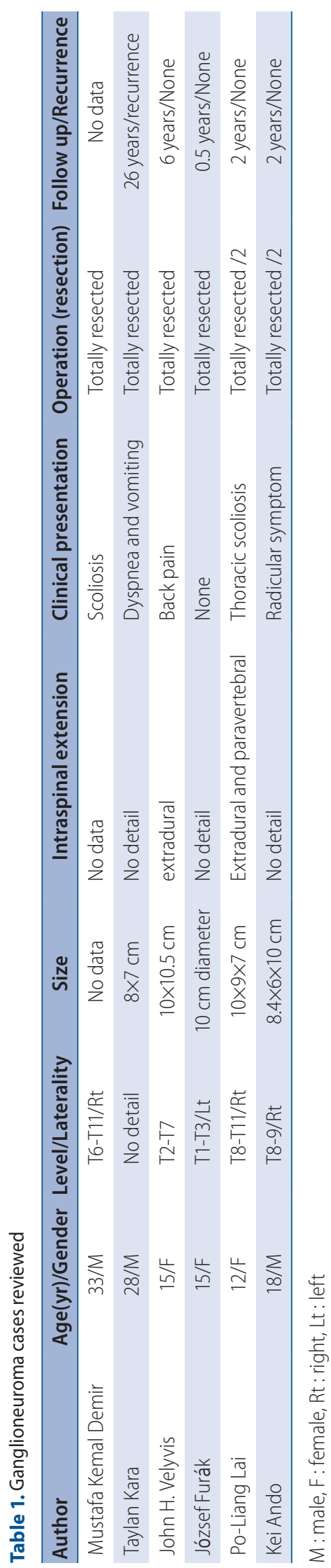

\section{DISCUSSION}

About $10 \%$ of GN may involve the spinal canal $^{4)}$. Paraspinal GN can extend into the spinal canal, forming dumb bell shaped tumor. However, in rare cases intradural extension has been reported. Most GNs are incidentally detected, and the symptoms, if any, are usually due to the mass effect. Rarely, the tumor may secrete vasoactive intestinal polypetide, resulting in diarrhea. As this slow growing tumor extends through the neural foramen into the spinal cord, some patients may present with neurological deficits or scoliosis ${ }^{2)}$.

Thoracic intradural extramedullary GNs are very rare, with only six cases reported so far to the best of our knowledge (Table 1). In our patient, the characteristic feature was the remarkably large tumor size infiltrating into the thoracic cavity along with an intradural component. Microscopically, these tumors contain large ganglion cells and show areas with smaller lymphocyte-like cells within a matrix of fibrous stroma and schwann cells. The distinction from malignant tumor is based on the absence of necrosis or presence of any immature ganglion cells ${ }^{7)}$.

It is usually safe and feasible to perform complete excision of GN. However, in case of multiple and/or large-sized tumors, multi-stage dissection should be considered. In the present case, there were dense adhesions of the tumor with the nerve roots at the foraminal portions, which were left undisturbed during dissection. GN generally has a favorable prognosis given its low metastatic potential ${ }^{5}$.

\section{CONCLUSION}

This report describes a rare case of multiple GNs of the thoracic spine with intradural extension. Ganglioneuromas should be considered in the differential diagnosis of a paraspinal mass. Although complete surgical resection is the best treatment option, stage-wise surgical resection should be considered in large-sized and/or multiple tumors, with close follow up.

\section{References}

1. Hioki A, Miyamoto K, Hirose Y, Kito Y, Fushimi K, Shimizu K : Cervical 
symmetric dumbbell ganglioneuromas causing severe paresis. Asian Spine J $8:$ 74-78, 2014

2. Kara T, Oztunali $C$ : Radiologic findings of thoracic scoliosis due to giant ganglioneuroma. Clin Imaging 37 : 767-768, 2013

3. Kyoshima K, Sakai K, Kanaji M, Oikawa S, Kobayashi S, Sato A, et al. : Symmetric dumbbell ganglioneuromas of bilateral C2 and C3 roots with intradural extension associated with von Recklinghausen's disease: case report. Surg Neurol 61 : 468-473; discussion 473, 2004

4. Pang BC, Tchoyoson Lim CC, Tan KK : Giant spinal ganglioneuroma. J Clin Neurosci 12 : 967-972, 2005

5. Son DW, Song GS, Kim YH, Lee SW : Ventrally located cervical dumbbell ganglioneuroma producing spinal cord compression. Korean J Spine $10: 246-248,2013$

6. Tsai FJ, Kuo KL, Tzou RD, Cheng YH, Hwang SL, Lieu AS : A huge extradural ganglioneuroma of the lumbar spine. Formosan J Surg 47 : 160165,2014

7. Ugarriza LF, Cabezudo JM, Ramirez JM, Lorenzana LM, Porras LF : Bilateral and symmetric C1-C2 dumbbell ganglioneuromas producing severe spinal cord compression. Surg Neurol 55 : 228-231, 2001

8. Vardas K, Manganas D, Papadimitriou G, Vougas V, Bakalis A, Chantziara $\mathrm{M}$, et al. : Presacral ganglioneuroma: diagnostic considerations and therapeutic strategy. Case Rep Oncol 6 : 561-568, 2013 